\title{
Millimeter-Wave InP Image Line Self-Mixing Gunn Oscillator
}

\author{
SAMUEL DIXON, JR., SENIOR MEMBER, IEEE, AND HAROLD JACOBS, FELLOW, IEEE
}

\begin{abstract}
Indium Phosphide Gunn Diodes have been used to develop self-mixing oscillators in the $60-\mathrm{GHz}$ region using dielectric image line techniques. Experiments have been performed to measure conversion characteristics and minimum detectable signal. Applications are suggested based on minimum detectable signal for systems requiring small size and low cost.
\end{abstract}

\section{INTRODUCTION}

T HE millimeter-wave image guide self-mixing oscillator is potentially a key element for future low-cost receiver front end designs. The main reason for this is that the self-mixing approach embodies simplifications in circuitry for the entire receiver system. Schottky barrier and other rectifier diodes suffer from the disadvantage of having a separate local oscillator and low burnout power limit. Bulk-effect Gunn self-oscillating mixers offer less sensitivity at $60 \mathrm{GHz}$, but have the attractive alternative of a high-power handling capability.

In conventional mixers, there usually exists a signal frequency, mixer diodes of rectifier type and a separate local oscillator. In the self-oscillating mixer, the mixer diode is eliminated. The Gunn diode will serve both as a local oscillator, and because nonlinearities are always present in an oscillator, as a mixing element. With the Gunn diode oscillator serving both these functions, the integrated receiver front-end design using the dielectric image guide approach becomes extremely compact and simplified. In the latter arrangement, the signal is fed directly into the oscillator and a suitable IF probe will remove the IF power for use in subsequent amplifier stages.

One of the objectives of this paper, was the design of self-oscillating mixers with considerable simplifications, and hence, reduction in cost. In the quest for lower cost, the image guide technology was applied using a Gunn diode in a simply constructed dielectric cavity. The InP Gunn diode was imbedded in an aperture which was cut in a high resistivity aluminumi oxide $\left(\mathrm{Al}_{2} \mathrm{O}_{3}\right)$ ceramic waveguide. The significance of the image line technology is that active devices, as well as passive components, can be developed and integrated into circuit modules to construct functional subsystems.

Manuscript received November 24, 1980; revised February 18, 1981. The authors are with the Millimeter Wave Devices and Circuits Team, U.S. Army Electronics Technology and Devices Laboratory, Fort Monmouth, NJ 07703.

\section{Device Design}

The Gunn oscillator cavity design is based on an imageline concept first formulated by Marcatili [1] and later modified for millimeter waves [2]. The fundamental electromagnetic wave propagating in an image guide is the $E_{11}^{y}$ mode, a hybrid mode which propagates when correctly launched. Application of theoretical considerations indicated that the image guide for proper operation should be on the order of one wavelength in the medium in width, and less than one-half wavelength in height. At $60 \mathrm{GHz}$, cross-sectional dimensions of the image guides were oversized, i.e., slightly greater than $1 \mathrm{~mm}$ in height and about 2 $\mathrm{mm}$ in width. Experiments indicated that in this oversized condition, the $E_{11}^{y}$ mode dominated. The resonant section in back of the Gunn diode was approximately $(2 N+1) \lambda / 2$ in length. A diagram of the image guide self-oscillating mixer is shown in Fig. 1. This simplified schematic indicates the manner of coupling to the metal waveguide showing one end of the resonant cavity being tapered. This taper can effect a low-loss match to the metal waveguide by sliding the tapered end into the metal waveguide for maximum power transfer. This matched condition also yielded optimum IF output when the RF input signal was introduced. Fig. 2 shows a more detailed cutaway view of the device investigated. As can be seen, the signal power propagates down the dielectric from the left with the IF output being extracted out the top of the image guide. A metal disk is being utilized as a tuning element for the Gunn diode. Fig. 3 shows an exploded view of the $60-\mathrm{GHz}$ image waveguide self-oscillating mixer which utilizes a tunable short to optimize performance. The metal housing was designed to support the biasing structure and the dimensions were such that it just covered the immediate area of the Gunn diode. The dielectric waveguide was exposed for most of its length, and experiments were not made on the unit without the dielectric. The Gunn diode is mounted flush with the bottom of the metal structure. The $\mathrm{Al}_{2} \mathrm{O}_{3}$ image waveguide with tapered front end was bonded to the metal housing, using a modified silver conductive adhesive, in such a way that the Gunn diode top protruded up into the dielectric. The dimensions of the image dielectric guide was 0.060 by 0.120 in. A 0.045 -in hole in the dielectric allowed the IF and bias voltage post to come down and make a pressure contact with the top of the Gunn diode. This method of applying the bias voltage 


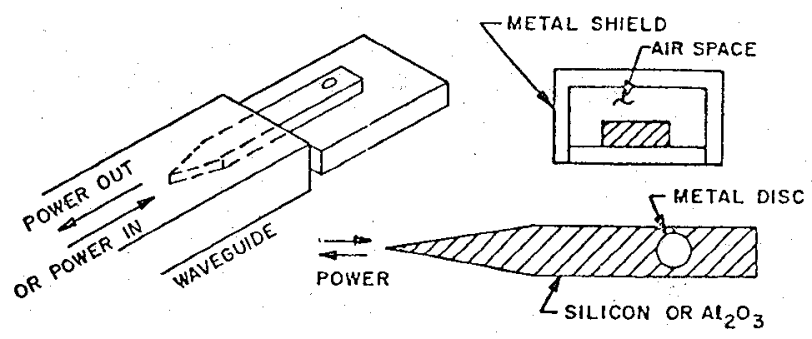

Fig. 1. Image guide self-oscillating mixer coupling to waveguide.

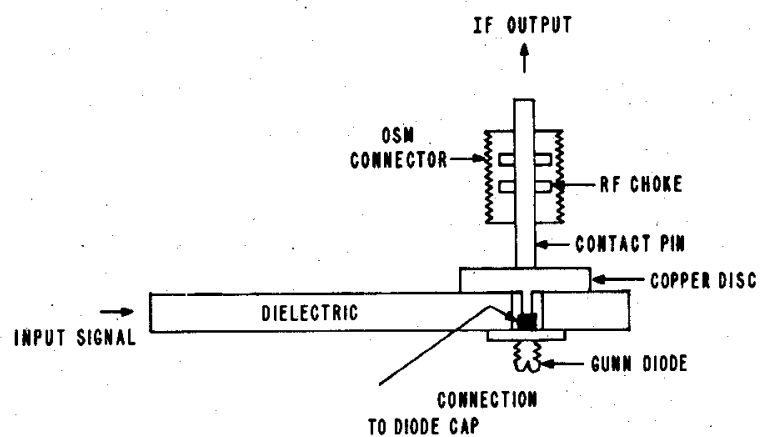

Fig. 2. Cutaway view of image guide self-oscillating mixer.

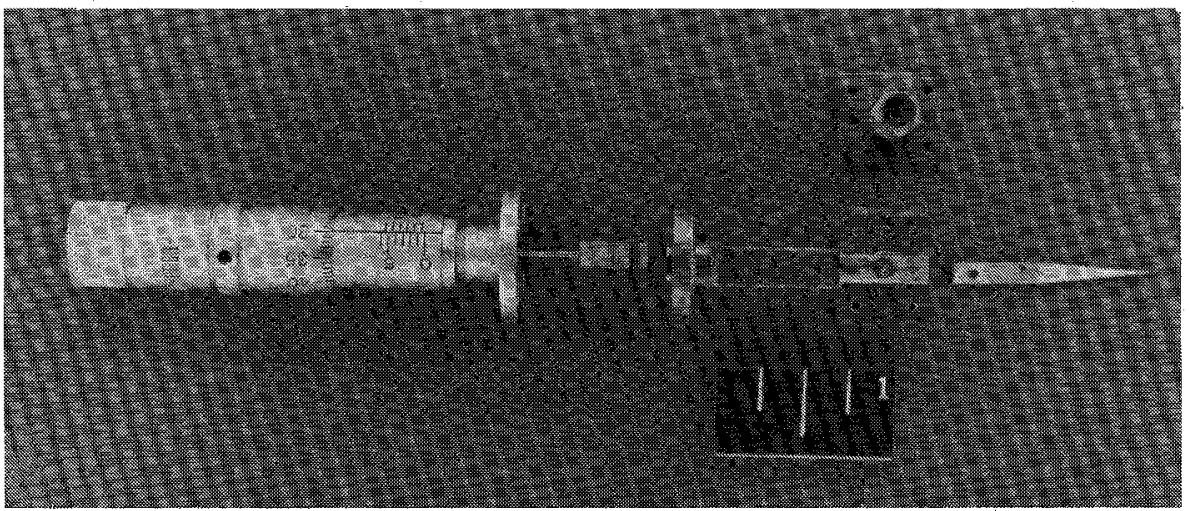

Fig. 3. Exploded view of the image guide self-oscillating mixer.

made it possible to mount a tuning short behind the image guide.

\section{EXPERIMENTAL RESULTS}

Fig. 4 shows the output power and frequency characteristics of the InP Gunn diode self-oscillating mixer as a function of the bias voltage. Fig. 5 shows the circuit used in the evaluating of the InP self-oscillating mixer. A wide-band (50 to $75 \mathrm{GHz}$ ) backward wave oscillator in a manual tuning mode was used as a signal source. The single frequency outputs from the source was stable within \pm 0.001 percent with nonharmonic spurious signals that are $40 \mathrm{~dB}$ down. This stable signal source was tuned $300 \mathrm{MHz}$ above or below the InP Gunn oscillator to produce an IF falling into the filter passband. All measurements were made in an IF noise bandwidth of $100 \mathrm{MHz}$. The amplitude of the IF power in $\mathrm{dBm}$ was compared with measured values of input signal power for conversion gain or loss measurements. Fig. 6 shows the conversion loss or gain as a function of the bias voltage. The minimum detectable signal is the principle parameter for determining the sensitivity of the self-mixing oscillator, that is, how weak a signal the device can detect. To determine the minimum detectable signal (MDS), the IF power, as viewed on the wide-band scope (500-MHz bandwidth), is decreased by placing attenuation in the signal channel. This IF energy was set equal to the noise level. At this point, the signal level is equal to the noise level and this signal power is defined as the minimum detectable signal measured in decibels referred to $1.0 \mathrm{~mW}(\mathrm{dBm})$. Fig. 7 shows the minimum detectable signal in a $100-\mathrm{MHz}$ bandpass as a function of bias voltage. As can be seen, the InP Gunn diodes MDS varies from -79 to $-55 \mathrm{dBm}$ as the bias voltage is varied from 7.3 to $8.1 \mathrm{~V}$. The MDS and conver- 


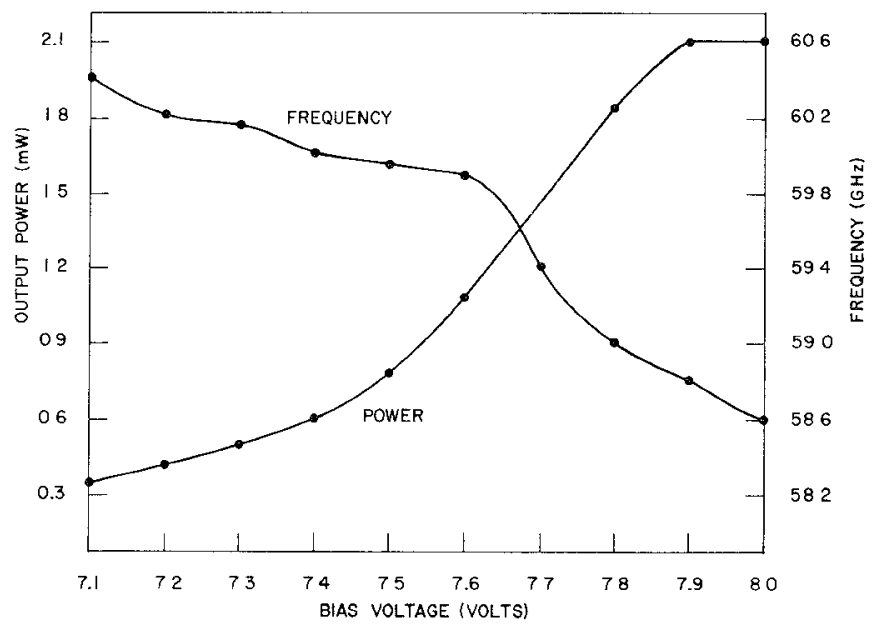

Fig. 4. Power/frequency characterıstics of InP Gunn oscillator.

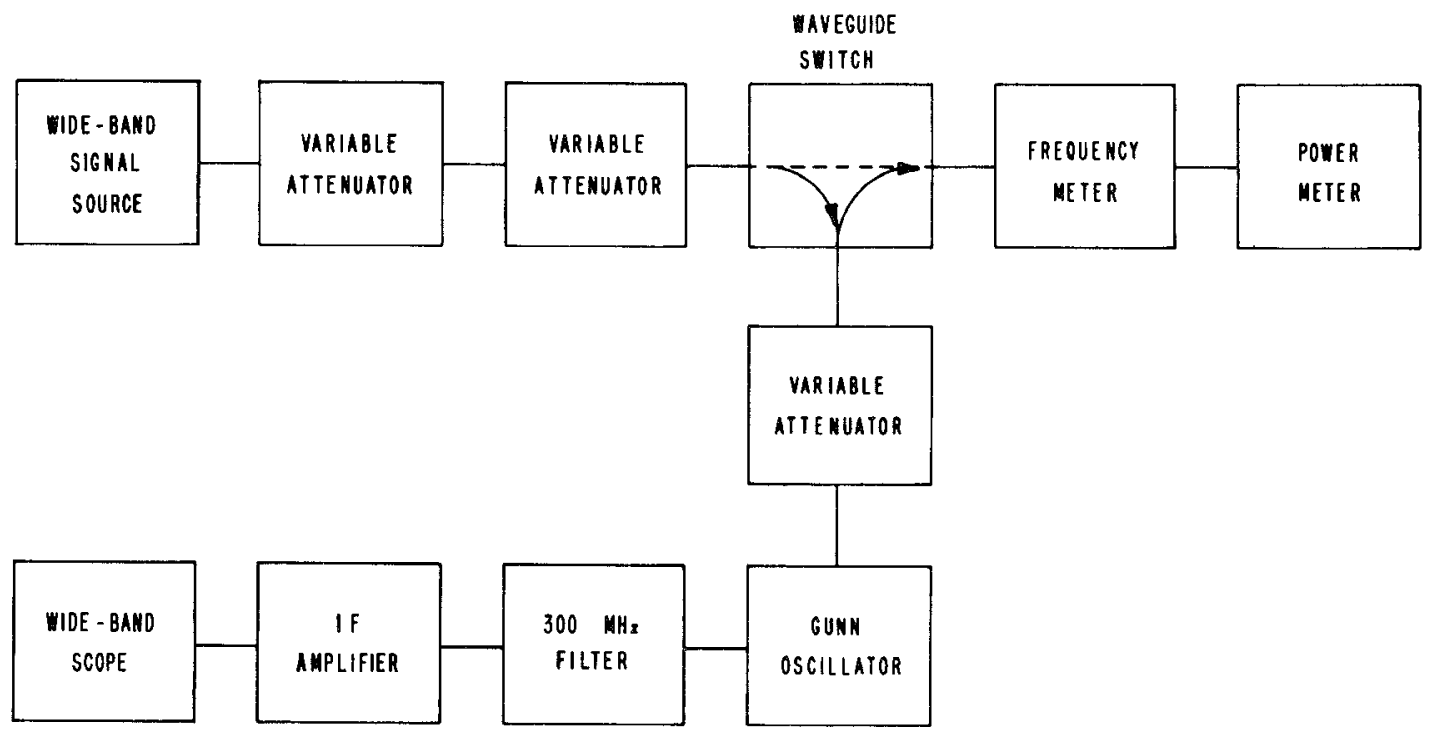

Fig. 5. Experimental setup for Gunn characterization.

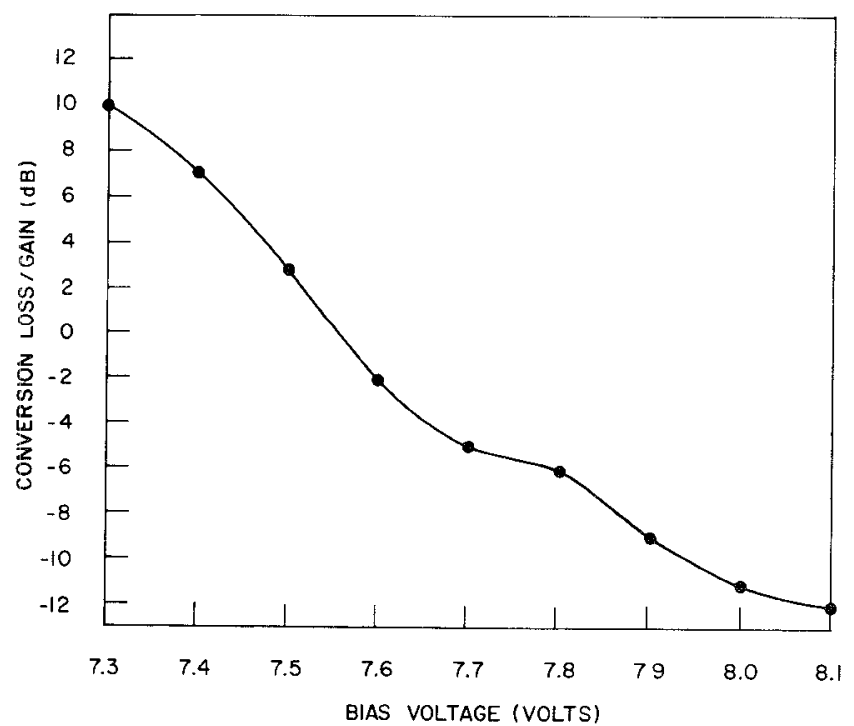

Fig. 6. Self-mixing oscillator conversion characteristics.

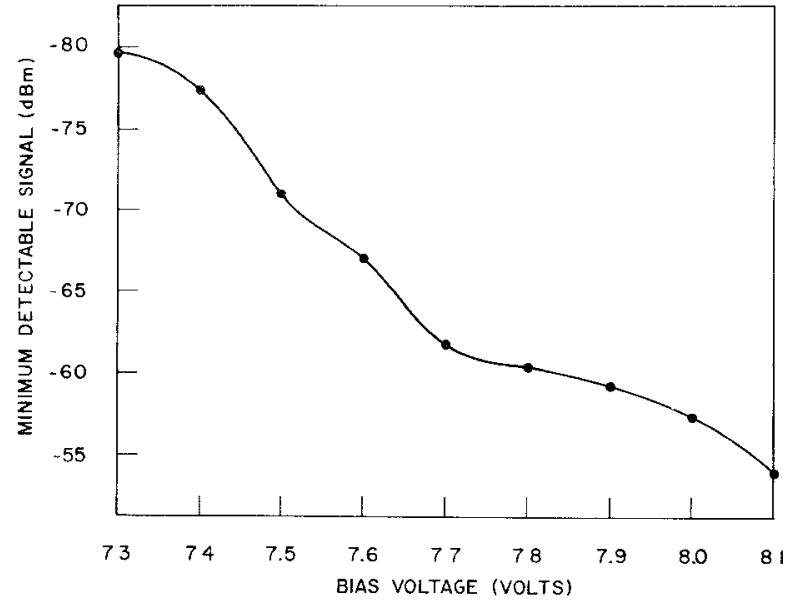

Fig. 7. Minimum detectable signal as a function of bias voltage. 
sion characteristic peak at a voltage just above threshold for oscillation. The conversion gain characteristic tends to be slightly unstable at this point and better operation can be attained at a higher bias voltage. This behavior is consistent with information reported previously by investigators at lower frequencies of operating using GaAs Gunn diodes in waveguide cavities [3]-[5].

The InP Gunn diode has emerged as a potentially lower noise device, and hence higher sensitivity self-mixing oscillator, than those utilizing GaAs material. The larger peakto-valley ratio of InP indicates higher electron velocity for a given field and leads to greater operating efficiency than GaAs. It is believed that the various scattering mechanisms which limit the high-frequency performance of transferred electron devices predict higher frequency operations for InP than GaAs [6].

\section{CONClusion}

It has been shown that, self-oscillating mixers using InP Gunn diodes can be successfully designed with low cost and simplified construction techniques. These devices are very lightweight and lend themselves to applications in integrated circuit modules and subassemblies. Experimental data indicate a peak sensitivity in the order of -79 $\mathrm{dBm}$ which is lower than a conventional Schottky barrier balanced mixer. However, the image waveguide device has the advantages of having a simplified construction with a high-signal power burnout level coupled with low unit cost. These characteristics make the image waveguide self-mixing oscillator, a viable device in low-cost receivers, expendable EW sensors, and short range terminal guidance. In addition, when compared with GaAs Gunn diodes, the InP self-mixing device has greater potential in the higher millimeter-wave frequency region (above $100 \mathrm{GHz}$ ) due to $\operatorname{InP}$ having a higher effective transit velocity and fast intervalley scattering [6], [7].

\section{REFERENCES}

[1] E. J. Marcatili, "Dielectric rectangular waveguide and directional couplers for integrated optics," Bell Syst. Tech. J., vol. 48, pp. 2017-2102, Sept. 1979.

[2] H. Jacobs, G. Novick, C. M. LoCasia, and M. M. Chrepta, "Measurement of guide wavelength in dielectric rectangular waveguide," Proc. IEEE, vol. MTT-24, pp. 812-815, Nov. 1976.

[3] M. J. Lazarus, S. Novak, and E. D. Bullimore, "New millimeter-wave receiver using self-oscillating Gunn diode mixers," Microwave J., vol. 14 , no. 7 , pp. $43-45$, July 1971 .

[4] M. J. Lazarus and S. Novak, "Millimeter-wave Gunn mixer with $-90 \mathrm{dBm}$ sensitivity, using a MOSFET/bipolar AFC circuit," Proc. IEEE, vol. 60, p. 747, June 1972.
[5] M. Kotani and S. Mitsui, "Self-mixing effect of Gunn oscillator," Electron. Comm. Jap., vol. 55-B, no. 12, pp. 60-67, 1972.

[6] F. A. Myers, "Efficient InP Gunn diodes shrink power requirements," Microwaves, May 1980.

[7] J. D. Crowley, S. B. Hyder, J. J. Sowers, "Millimeter-wave indium phosphide Gunn devices," R\&D Tech. Rep. DELET-TR-2940, June 1979.

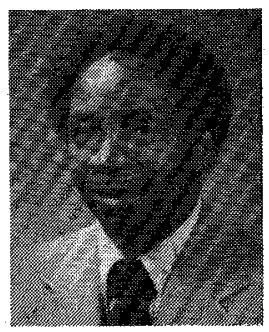

Samuel Dixon, Jr. (SM'74) received the B.S. degree in physics from West Virginia State College, Institute, WV, in 1950, and the M.S. degree in electronic engineering from Monmouth College, West Long Branch, NJ, in 1972.

After serving three years in the U.S. Army Field Artillery as a First Lieutenant, he joined the U.S. Army Signal School as a Radar Instructor in 1955. He transferred to the U.S. Army Electronics Laboratories in 1958. Since that time he has been involved in research and development programs involving solid state ferrite and semiconductor components for microwave and millimeter-wave radar, communications, and electronic-warfare systems. He is presently with the Electronic Technology and Devices Laboratory of the Electronic Research and Development Command, Fort Monmouth, NJ.

Mr. Dixon is a member of Eta Kappa Nu and Sigma Pi Sigma.

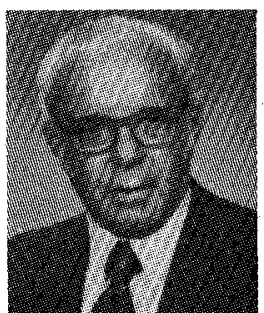

Harold Jacobs (SM'59-F'68) was born in Port Chester, NY, on November 21,1917 . He received the B.A. degree from The John Hopkins University, Baltimore, MD, and the M.S. and Ph.D. degrees from New York University, New York, NY.

He joined the U.S. Army Signal Corps Laboratory, Fort Monmouth, NJ, in 1949, with previous experience at RCA Mgn. Company, Lancaster, PA, and Sylvania Electric Products, Kew Gardens, NY. He has worked in the areas of electron tubes, solid-state devices, lasers, and microwave and millimeterwave devices. He has served as Professor of Electronic Engineering at Monmouth College, West Long Branch, NJ.

Dr. Jacobs received the IEEE Fellow Award in 1967 for his semiconductor devices contributions and the Army's Decoration for Exceptional Civilian Service in 1969 for millimeter-wave imaging investigations. In 1973 he was the recipient of the IEEE's Harry Diamond Award for identification of bulk semiconductor effects at millimeter waves with applications to imaging and surveillance. 